Supporting Information for:

\title{
Determining the genetic regulation and coordination of lignification in stem tissues of Arabidopsis using semi-quantitative Raman microspec- troscopy
}

Leonard Blaschek $^{1 \dagger}$, Nuoendagula ${ }^{2 \dagger}$, Zoltán Bacsik ${ }^{3}$, Shinya Kajita ${ }^{2}$ and Edouard Pesquet ${ }^{1 \star}$

${ }^{1}$ Arrhenius laboratories, Department of Ecology, Environment and Plant Sciences (DEEP), Svante Arrhenius väg 20A, Stockholm University, 10691 Stockholm, Sweden

${ }^{2}$ Graduate School of Bio-Applications and Systems Engineering, Tokyo University of Agriculture and Technology, Tokyo 184-8588, Japan

${ }^{3}$ Arrhenius laboratories, Department of Materials and Environmental Chemistry (MMK), Svante Arrhenius väg 16C, Stockholm University, 10691 Stockholm, Sweden

* corresponding author (edouard.pesquet@su.se)

${ }^{\dagger}$ contributed equally

The following 7 figures and one table are presented on a total of 9 pages:

Figure S1 Raman spectra of all measured monomeric lignin model compounds.

Figure S2 Raman spectra of polymeric lignin model compounds acquired using a $785 \mathrm{~nm}$ laser.

Figure S3 Loading plots of the PCA separating different stem tissues based on their Raman spectra.

Figure S4 Relationship between the area under the curve (AUC) of Raman spectra and the measured cell wall area.

Figure S5 Close up of the $1550-1700 \mathrm{~cm}^{-1}$ region in the different mutants.

Figure S6 Correlations and linear regressions of non-normalized Raman band intensities with lignin composition data from pyrolysis-GC/MS.

Figure S7 Correlations and linear regressions of Raman band intensities normalized to the $1603 \mathrm{~cm}^{-1}$ band with lignin composition data from pyrolysis-GC/MS.

Table S1 Relative intensity of Raman scattering and autofluorescence in the different monomeric and polymeric lignin model compounds. 


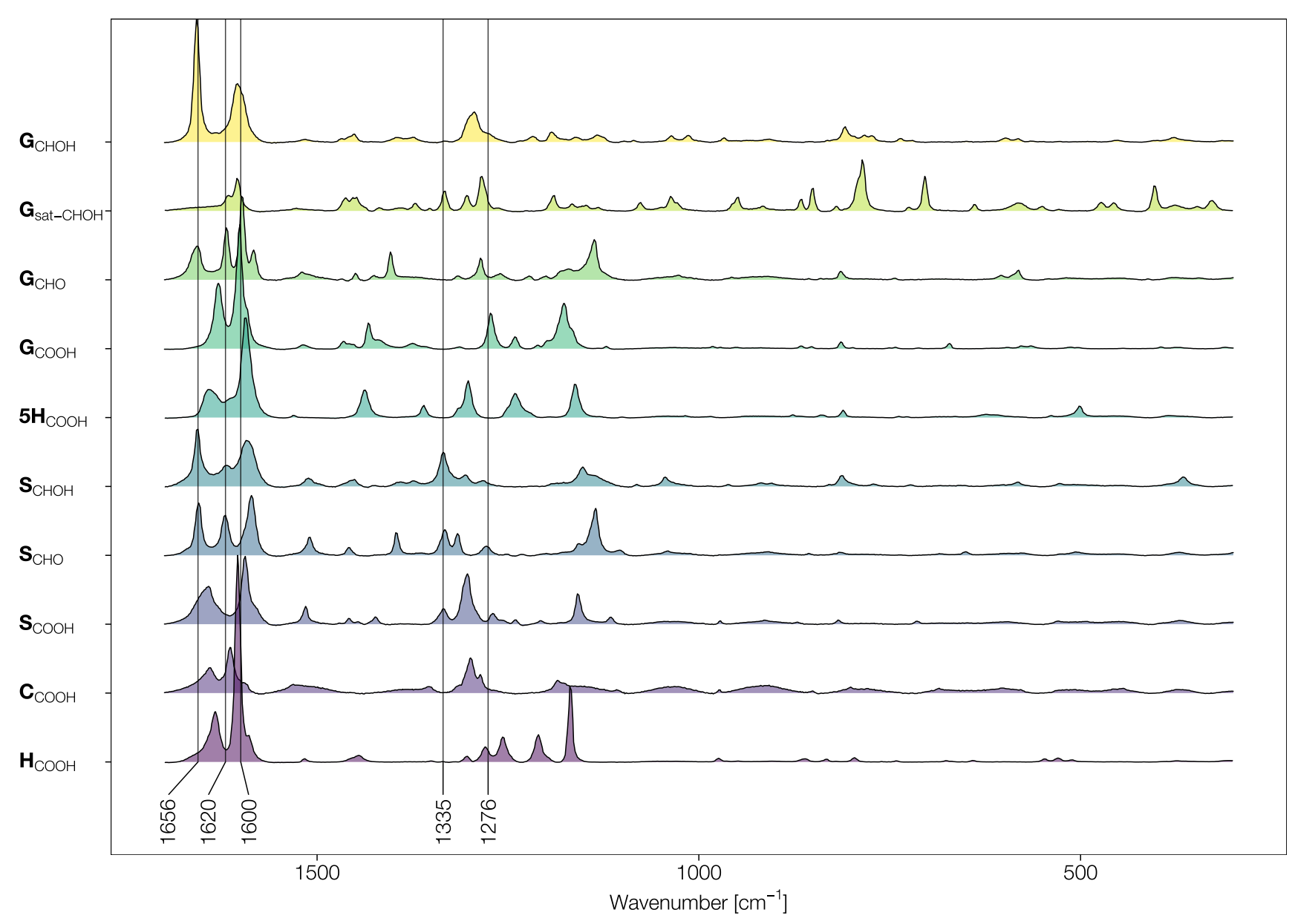

Figure $\mathbf{S 1}$ | Spectra of all measured monomeric lignin model compounds. Spectra were baseline corrected and their intensity at each wavenumber was divided by the total Raman signal of the respective compound (area under the curve, AUC) between 300 and $1700 \mathrm{~cm}^{-1}$. This scaling method is hereafter referred to as normalization to the AUC.

$\mathbf{G}_{\mathrm{CHOH}}$, coniferyl alcohol; $\mathbf{G}_{\mathrm{sat}-\mathrm{CHOH}}$, dihydroconiferyl alcohol; $\mathbf{G}_{\mathrm{CHO}}$ coniferaldehyde; $\mathbf{G}_{\mathrm{COOH}}$, ferulic acid; $\mathbf{5} \mathbf{H}_{\mathrm{COOH}}, 5$-hydroxyferulic acid; $\mathbf{S}_{\mathrm{CHOH}}$, sinapyl alcohol; $\mathbf{S}_{\mathrm{CHO}}$, sinapaldehyde; $\mathbf{S}_{\mathrm{COOH}}$, sinapic acid; $\mathbf{C}_{\mathrm{COOH}}$, caffeic acid; $\mathbf{H}_{\mathrm{COOH}}, p$-coumaric acid. 


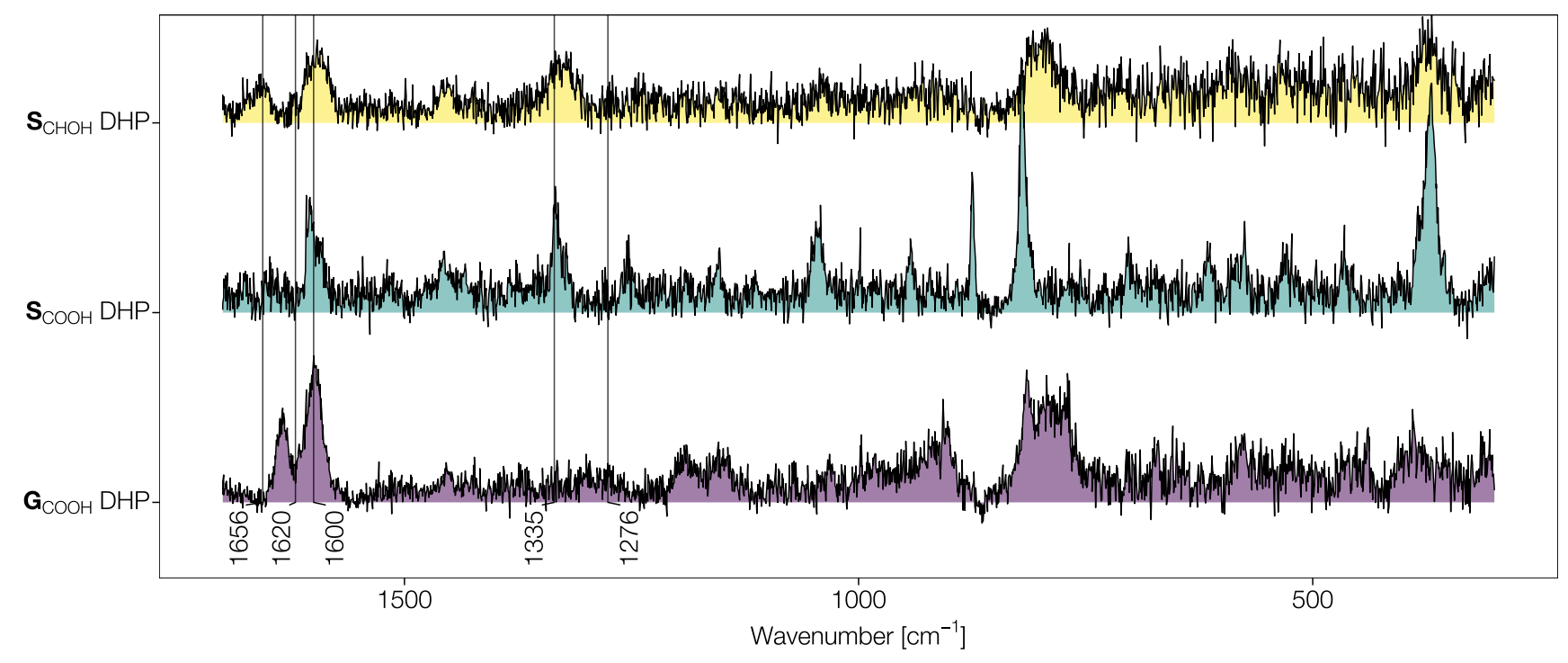

Figure S2 | Spectra of DHPs that were not measurable with the $532 \mathrm{~nm}$ laser acquired using a $785 \mathrm{~nm}$ laser, normalized to their AUC. 
A

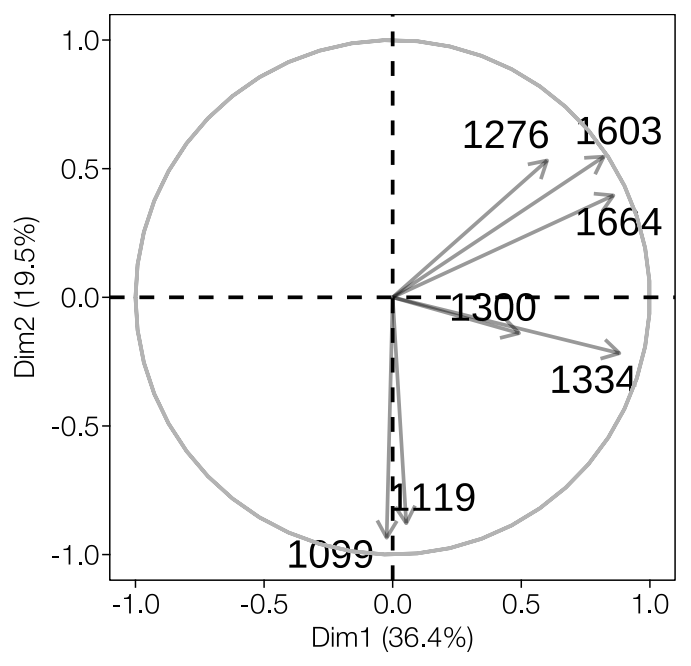

B

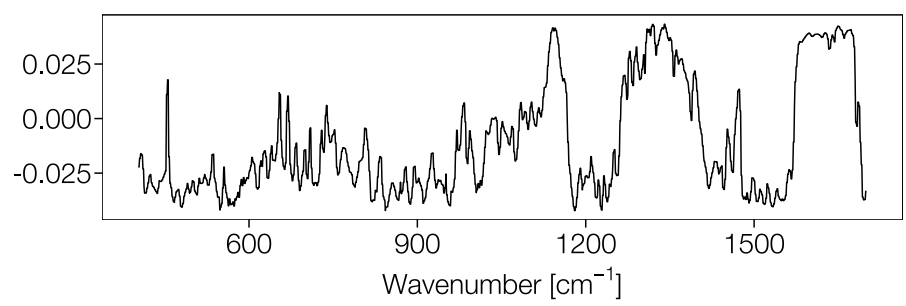

C PC2

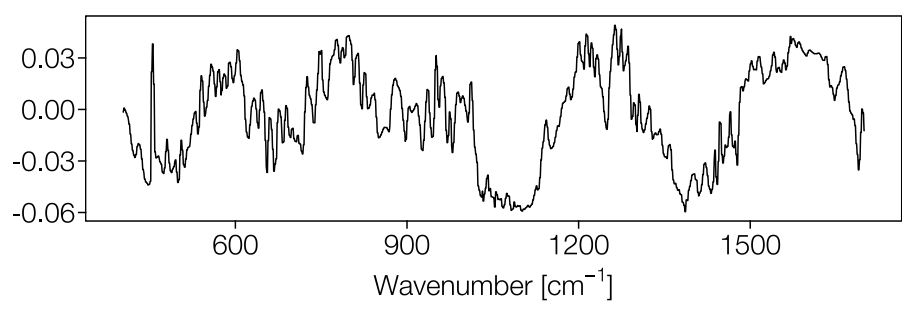

Figure S3 | Loading of the PCA shown in figure 2B.

A contributions of characteristic Raman bands. Apart from the typical cell wall polymer bands (see table 1), the band at $1300 \mathrm{~cm}^{-1}$ is characteristic for saturated waxes in the cuticle (Prats Mateu et al. 2016).

B Loading of principle component (PC) 1 for each wavenumber.

C Loading of PC2 for each wavenumber. 
A

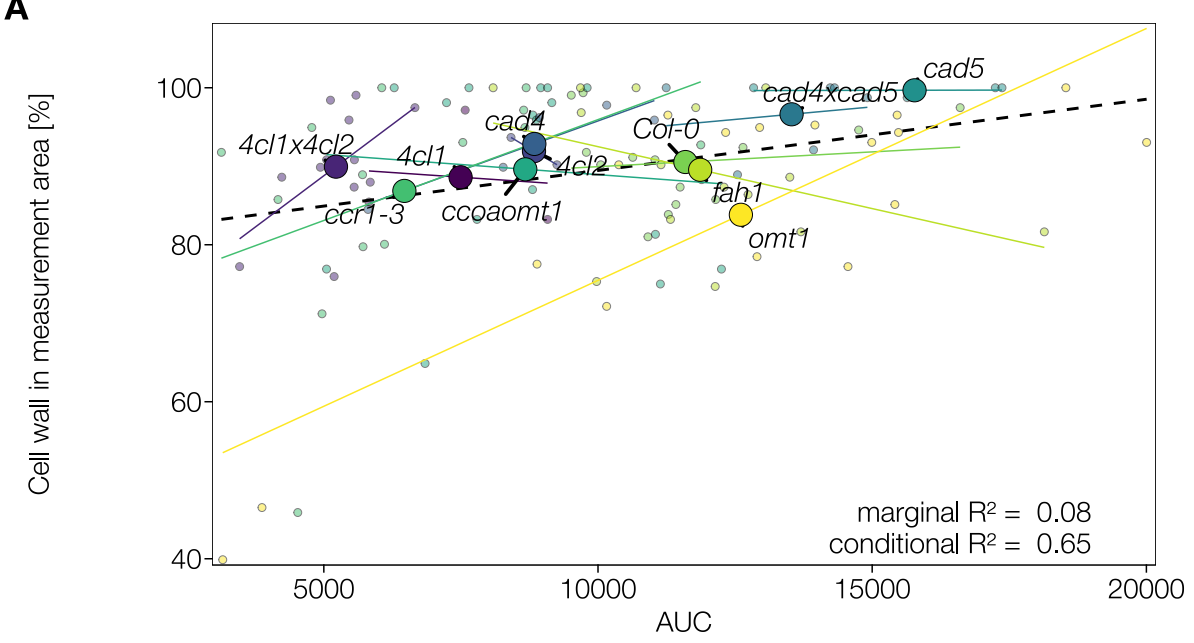

B

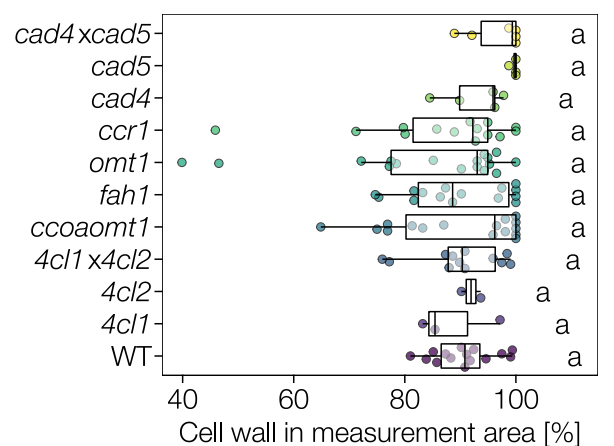

C

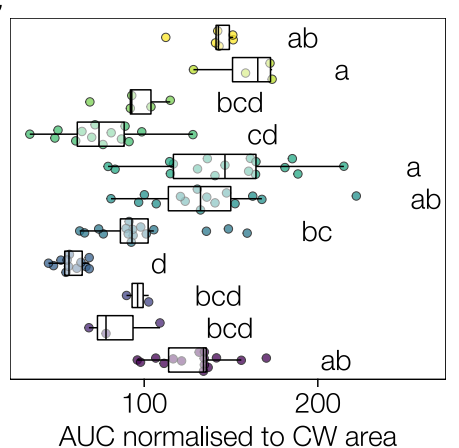

Figure S4 | Relationship between AUC and measured cell wall area.

A Linear regressions across genotypes (dashed line) and within genotypes (coloured and labelled) for the AUC as a function of the cell wall coverage in the measurement area. Fitting a linear mixed model and allowing for genotype specific slopes and intercepts as random effects results in a marginal $R^{2}$ of 0.08 (variation explained purely by cell wall area) and a conditional $R^{2}$ of 0.65 (including the random effects of the genotypes).

B Cell wall coverage of the measurement area in the different genotypes. Statistically significant differences are indicated by different letters (Tukey-HSD test , $\alpha=0.05)$.

C The AUC in the different genotypes normalized to their respective cell wall area from panel B. The variance shown is therefore purely due to different composition and/or orientation of the cell wall polymers. Statistically significant differences are indicated by different letters (Tukey-HSD test,$\alpha=0.05)$. 


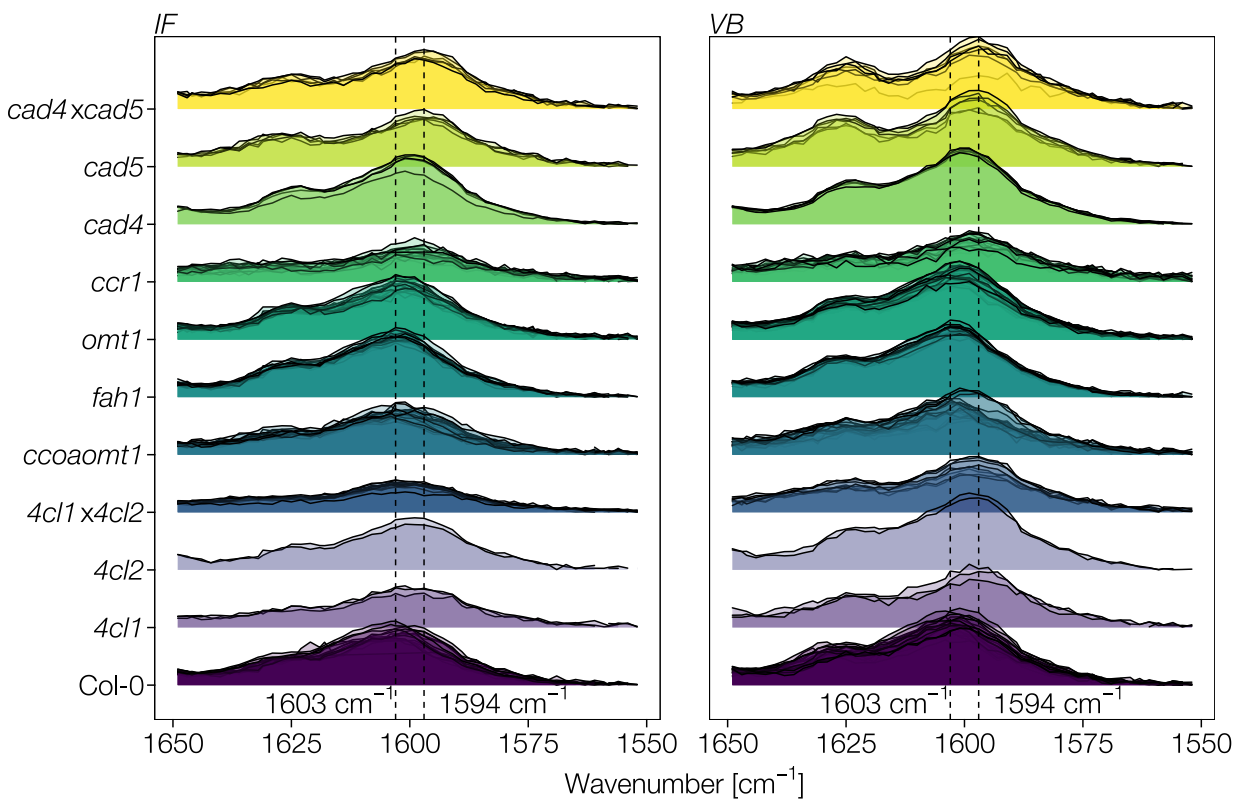

Figure S5 | Zoom of the mutant spectra on the aromatic region between 1550-1700 $\mathrm{cm}^{-1}$. Replicates are shown as individual semi-transparent spectra and overlayed by genotype. The range of the positional shift of the $\sim 1600 \mathrm{~cm}^{-1}$ band between genotypes is indicated. 


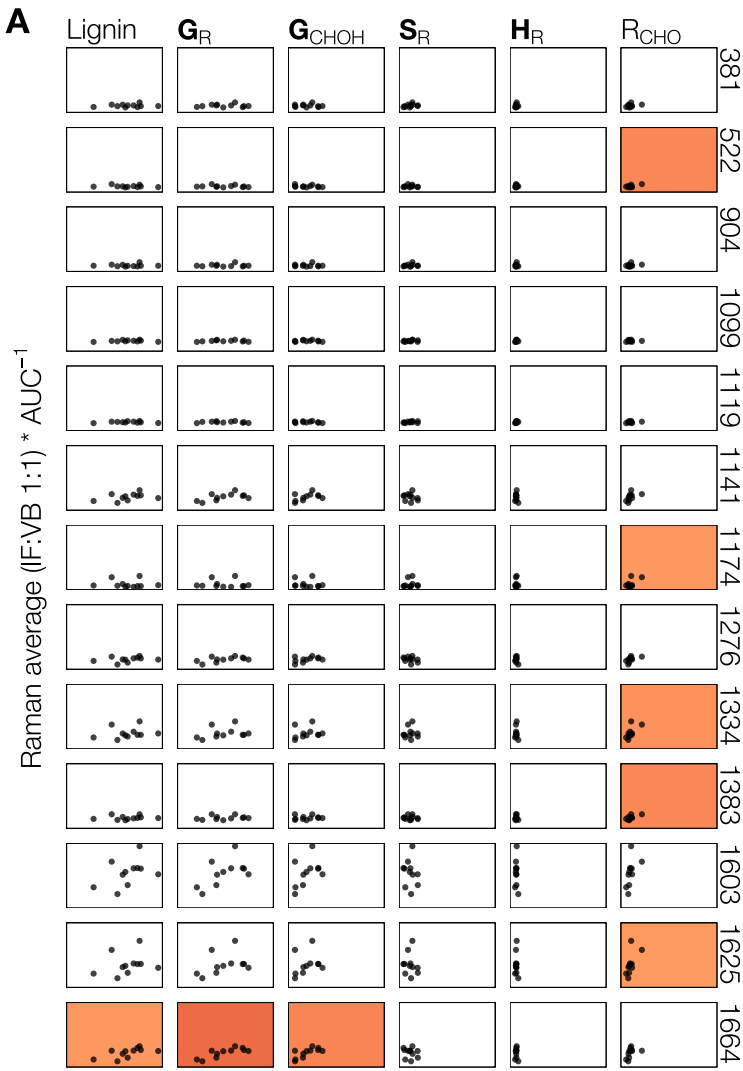

Pyrolysate * total pyrolysate ${ }^{-1}$

Pearson's $r$ (if $p<0.05$ )

\begin{tabular}{l|l|l|l|l|l}
-1.0 & -0.5 & 0.0 & 0.5 & 1.0
\end{tabular}
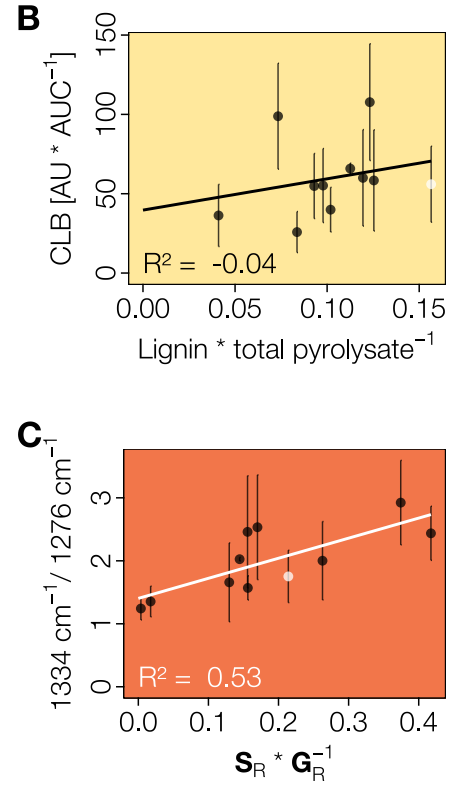

D

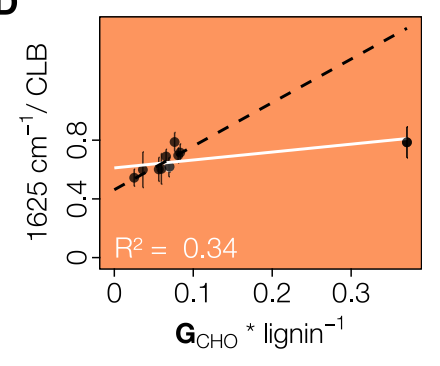

Figure S6 | Correlations and linear regressions of non-normalized Raman band intensities with lignin composition data from pyrolysis-GC/MS. A Correlation between band intensities of the respective spectrum and total pyrolysis GC/MS data.

B Correlation of the composite lignin band $\left(\mathrm{CLB}=\frac{\text { Intensity }_{1603 \mathrm{~cm}^{-1}}}{2}+\right.$ Intensity $\left._{\left.1334 \mathrm{~cm}^{-1}\right)}\right)$ with total lignin amount.

C Correlation of the ratio of band intensities at $1334 \mathrm{~cm}^{-1}{ }^{2}$ to $1276 \mathrm{~cm}^{-1}$ with the $\mathbf{S} / \mathbf{G}$ ratio.

D Correlation of the ratio of band intensity at $1625 \mathrm{~cm}^{-1}$ to $\mathrm{CLB}$ and $\mathbf{G}_{\mathrm{CHO}}$ content relative to total lignin. The dashed line represents the regression excluding cad4 $\times \operatorname{cad5}\left(R^{2}=0.55\right)$. 


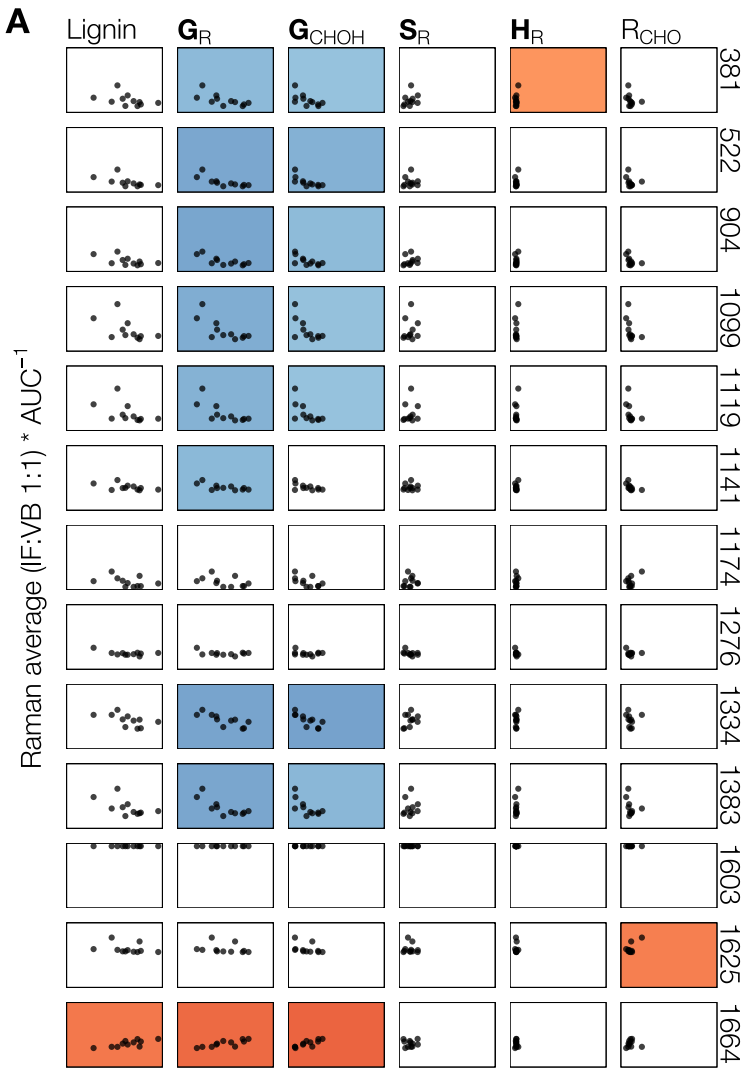

Pyrolysate * total pyrolysate ${ }^{-1}$

Pearson's $r$ (if $p<0.05$ )

\begin{tabular}{l|l|l|l|l|l|l|l}
-1.0 & -0.5 & 0.0 & 0.5 & 1.0
\end{tabular}
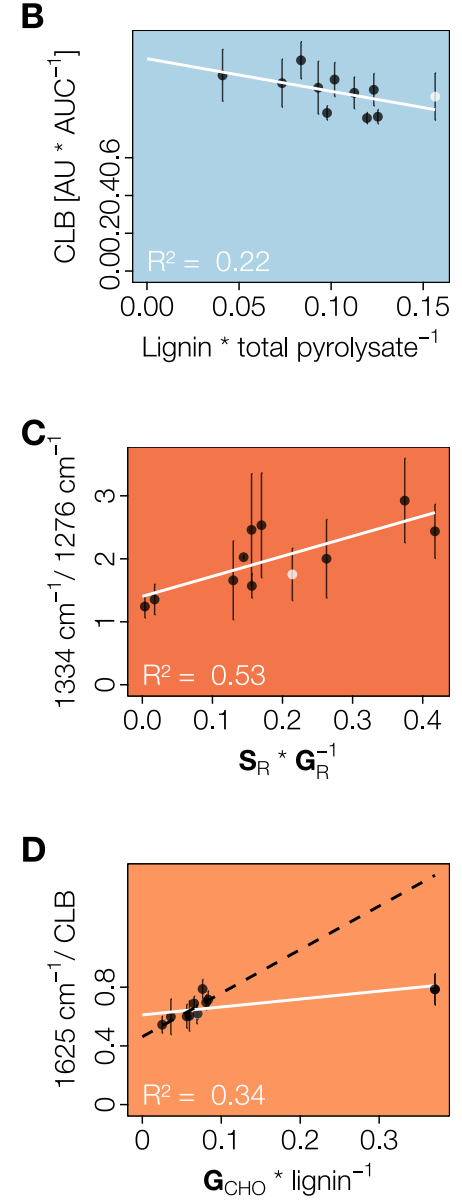

Figure S7 | Correlations and linear regressions of Raman band intensities normalized to the $1603 \mathrm{~cm}^{-1}$ band with lignin composition data from pyrolysisGC/MS.

A Correlation between band intensities normalized to the $1603 \mathrm{~cm}^{-1}$ band of the respective spectrum and total pyrolysis GC/MS data.

B Correlation of the composite lignin band (CLB $=\frac{\text { Intensity }_{1603 \mathrm{~cm}^{-1}}}{2}+$ Intensity $_{\left.1334 \mathrm{~cm}^{-1}\right)}$ normalized to the $1603 \mathrm{~cm}-1$ band with total lignin amount.

C Correlation of the ratio of band intensities at $1334 \mathrm{~cm}^{-1}{ }^{2}$ to $1276 \mathrm{~cm}^{-1}$ with the $\mathbf{S} / \mathbf{G}$ ratio.

D Correlation of the ratio of band intensity at $1625 \mathrm{~cm}^{-1}$ to $\mathrm{CLB}$ and $\mathbf{G}_{\mathrm{CHO}}$ content relative to total lignin. The dashed line represents the regression excluding cad $4 \times \operatorname{cad} 5\left(R^{2}=0.55\right)$ 
Table S1 | Relative intensity of Raman scattering and autofluorescence in the different monomeric and polymeric lignin model compounds.

\begin{tabular}{|c|c|c|c|c|}
\hline \multirow[b]{2}{*}{ Compound } & \multicolumn{2}{|c|}{ Relative AUC ${ }^{\mathrm{a}}$} & \multicolumn{2}{|c|}{ Signal $_{\text {Raman }}{ }^{*}$ Signal $_{\text {fluo }}{ }^{-1}$} \\
\hline & Monomeric & DHP & Monomeric & DHP \\
\hline $\mathrm{G}_{\mathrm{CHOH}}$ & 1.00 & 0.21 & 0.08 & 0.02 \\
\hline $\mathrm{G}_{\mathrm{sat}-\mathrm{CHOH}}$ & 0.84 & - & 0.05 & - \\
\hline $\mathrm{G}_{\mathrm{CHO}}$ & 2.10 & 0.58 & 0.04 & 0.03 \\
\hline $\mathrm{G}_{\mathrm{COOH}}$ & 1.45 & - & 0.16 & - \\
\hline $5 \mathbf{H}_{\mathrm{COOH}}$ & 4.09 & - & 0.08 & - \\
\hline $\mathrm{S}_{\mathrm{CHOH}}$ & 0.12 & - & 0.05 & - \\
\hline $\mathrm{S}_{\mathrm{CHO}}$ & 0.24 & 0.14 & 0.05 & 0.03 \\
\hline $\mathrm{S}_{\mathrm{COOH}}$ & 0.20 & - & 0.04 & - \\
\hline $\mathrm{C}_{\mathrm{COOH}}$ & 8.15 & - & 0.02 & - \\
\hline $\mathbf{H}_{\mathrm{COOH}}$ & 0.74 & - & 0.13 & - \\
\hline
\end{tabular}

${ }^{a}$ Relative to the AUC of monomeric $\mathrm{G}_{\mathrm{CHOH}}$ 